\title{
Mobile Technology to Monitor Physical Activity and Wellbeing in Daily Life Objective and Subjective Experiences of Older Adults
}

\author{
Miriam Cabrita $^{1,2}$, Monique Tabak ${ }^{1,2}$ and Miriam Vollenbroek-Hutten ${ }^{2}$ \\ ${ }^{1}$ Roessingh Research and Development, Telemedicine group, Enschede, Netherlands \\ ${ }^{2}$ Faculty of Electrical Engineering, Mathematics and Computer Science, Telemedicine group, \\ University of Twente, Enschede, Netherlands
}

Keywords: Wearables, Active Ageing; Positive Emotions.

Abstract: Older adults are not reaching the recommended guidelines for physical activity. There is growing evidence that physical activity and positive emotions reinforce each other. However, the development of interventions leveraging this knowledge faces several challenges, such as the limited knowledge on the assessment of emotional wellbeing in daily life using technology. In this study, we investigate the experience of older adults regarding the use of mobile technology to coach physical activity and monitor emotional wellbeing during one month. Our results show that the participants became more aware of their daily physical activity and perceived an added value in using the technology in daily life. However, only limited added-value was perceived on monitoring positive emotions in daily life in the way we performed it. The most common argument concerned repetitiveness of the questions being asked every day. Moreover, participants also reported that they were not used to think about their emotions, what affected the way they answered the questions regarding their emotional wellbeing. Our results suggest that, to ensure reliability of the data, it is extremely important to hear the experience of the participants after performing studies in daily life.

\section{INTRODUCTION}

Lack of physical activity and prevalence of physical inactive is a global problem. The World Health Organization points out physical inactivity as the fourth leading cause for global mortality (World Health Organization 2009). Despite the overall policies for promotion of physical activity and the well-known benefits for physical and mental health, older adults are still not active. In the literature, the proportion of older adults reaching the recommended guidelines ranges from 2 to $83 \%$, depending on the guidelines and assessment methods chosen (Sun et al. 2013). Mobile technology has already provided promising results in promoting physical activity, yet the older population shows low interest in using activity trackers (Alley et al. 2016). One reason might be that the market of physical activity trackers often targets a young, active and healthy population. It is therefore important to hear the experiences and opinions of the older adults, when intending to develop interventions to promote physical activity using mobile technology.
One emerging line of research combines promotion of healthy lifestyles with promotion of emotional wellbeing. For example, adapting Frederickson's 'upward spiral theory of lifestyle change' to the promotion of physical activity, being physically active might enhance emotional wellbeing and, in turn, higher experience of emotional wellbeing might motivate people to be more engaged and active (Fredrickson 2013). To be confirmed, this theory might open new horizons on interventions promoting physical activity and, furthermore, combining physical and mental health.

Mobile technology allows innovative methods to assess multiple parameters simultaneously. However, there is limited knowledge on the assessment of emotional wellbeing in daily life, especially using mobile technology. Emotional wellbeing concerns positive affective states, or positive emotions (Fredrickson 2001; Lyubomirsky et al. 2005). Experience sampling, also known as ecological sampling, is a commonly used method in research to assess emotions in daily life (Csikszentmihalyi \& Hunter 2003), and provides the means to assess 
positive emotions following the requirements proposed by Kanning and colleagues (Kanning et al. 2013). Despite its value for research, it has been less explored as a monitoring method, to be used over longer periods of time and create awareness about the own wellbeing.

In this study, we present the results of a onemonth daily life study that investigates objective and subjective experiences of community-dwelling older adults regarding physical activity promotion and monitoring of positive emotions in daily life. This work has two main objectives:

1 To investigate how older adults experience promotion of physical activity in their daily life using mobile technology;

2 To investigate how older adults experience monitoring positive emotions in their daily life using mobile technology;

As an exploratory study we also propose to investigate the relation between physical activity and experience of positive emotions per day.

This study is innovative as it provides older adults with technology that is normally more appealing to younger adults, and by investigating both objective and subjective experience of using this technology in daily life. This study focuses on the individual experience, following each participant for the course of one month with an interview at the end of this period to elicit subjective experiences. The results of this study will be used in the development of technology-based interventions to promote physical activity and emotional wellbeing among older adults.

\section{METHODS}

\subsection{Participants}

Twenty-three older adults were recruited in the PERSSILAA project (www.perssilaa.eu) and in events related to promotion of healthy behaviours. All who were interested received information letters via post explaining the research in more detail and were invited for an interview at the premises of the participating institution. Twelve older adults (7 female) accepted to participate in the study. Technology was explained by two researchers and the participants had time to ask questions. Participants were asked to use the technology at their own pace during four weeks. This study adhered to the guidelines set forth by the Declaration of Helsinki and it was approved by the institutional review board at the participating institution. All participants provided written informed consent.
The average age of the participants was 69 years old (range $65-78$ years). Three participants lived alone, 8 with someone else and 1 did not want to share this information. This participant dropped out after 5 days due to data privacy concerns. Although authorization was given to use the 5 days of data, we decided to not include the data. According to the frailty assessment from the Groningen Frailty Indicator (GFI) (Steverink et al. 2001), 3 participants suffered from decline, 7 were robust, and 2 were inconclusive due to missing data. In particular, 2 participants evidenced some physical function decline when assessed with the Short Form-36 Health Survey (Ware 1993). Table 1 provides a global overview of the demographics and health related characteristics of the participants.

Table 1: Characteristics of the participants $(n=11)$.

\begin{tabular}{ll}
\hline Characteristic & N, range, (\%) \\
\hline Age (mean, range) & $69,65-78$ \\
Gender & \\
$\quad$ Female & $6(55 \%)$ \\
Male & $5(45 \%)$ \\
Living Situation & \\
$\quad$ Alone & $3(27 \%)$ \\
With someone else & $8(73 \%)$ \\
Education & $1(9 \%)$ \\
$\quad$ Elementary School & $3(27 \%)$ \\
High School & $6(55 \%)$ \\
Vocational School & $1(9 \%)$ \\
$\quad$ University & $3(27 \%)$ \\
General Frailty (GFI) & $7(64 \%)$ \\
$\quad$ Decline & $1(9 \%)$ \\
Robust & \\
Missing & $2(18 \%)$ \\
Physical Function (SF-36) & $9(82 \%)$ \\
Decline & $25.3(17.4-36.1)$ \\
Robust & \\
BMI (mean, range) &
\end{tabular}

\subsection{Measurements}

Physical Activity. Physical activity was monitored using a Fitbit Zip ${ }^{\circledR}$ step counter that can be worn in the pocket to assess number of steps throughout the day. Literature has shown that this step counter provides a valid estimation of the number of steps in both the laboratory (Case et al. 2015; Lee et al. 2014; Kooiman et al. 2015; Nelson et al. 2016) and freeliving (Ferguson et al. 2015; Tully et al. 2014; Kooiman et al. 2015) environments with accuracy values ranging between 0.90 and 1 in both conditions. 
Feedback on physical activity was provided on the device itself, and also on the screen of the mobile phone, using the Activity Coach application. This application is a re-design of the application developed by Roessingh Research and Development and tested with several clinical populations, such as cancer survivors (Wolvers et al. 2015) and patients suffering from chronic pulmonary obstructive disease (Tabak et al. 2014). In the mobile phone, participants received feedback on the number of steps at that moment, number of steps at the current day per hour and during the last week per day. Participants could also see a representation of how far they were from reaching the daily goal. The daily step goal was set to 7500 steps, following the research of Tudor-Locke and colleagues (Tudor-Locke et al. 2011). Participants were told that this goal could be changed upon request.

Positive emotions. Emotional wellbeing was operationalized by 6 discrete positive emotions. Participants were asked at the end of every day (at 20:30) to which extent they experienced six discrete positive emotions (joy, amusement, awe, love/friendliness, interest and serenity) and to rate it on a Likert scale from 1 ('not at all') to 7 ('very intense'). The positive emotions asked were taken from the modified Differential Emotions Scale (Fredrickson 2013). The emotions were chosen to cover the full arousal, or activation, dimension.

Usability and feasibility. At the end of the 4-week period, participants were invited for a semi-structured interview to share their experience. The objective of this interview was to obtain an extended evaluation of the usability and feasibility of the system. Examples of questions asked were "Which features of the Activity Coach do you consider as the most important?" and "Did you become more aware of your wellbeing by answering the questions daily?".

\subsection{Data Analysis}

The interviews were audio recorded and transcribed verbatim. The transcripts were categorized in themes and sub-themes using inductive thematic analysis (Braun \& Clarke 2006). An iterative process was taken until eliciting the final codes.

Correlations between physical activity and positive emotions are calculated with bivariate correlation analysis. A composed variable of daily positive emotions was created by summing the results of the 6 emotions. Physical activity was operationalized in 4 discrete variables retrieved directly from the Fitbit classifications: number of steps per day, and number of minutes per day spend in each one of the following activity levels: inactive, lightly active, moderate-to-very active.

\section{RESULTS}

Eleven older adults participated in the study on an average of 27 days resulting on a total of 292 days of data collected. The daily average of steps was slightly above 6000 steps, with the daily averages among participants varying from 2989 to 10572 steps per day. Table 2 provides a summary of the combined results from all participants.

\subsection{Experience of Promotion of Physical Activity in Daily Life}

Figure 1 provides an overview of the number of steps per day performed by four participants. Only 1 participant in the study consistently met the daily goal, 5 participants almost never reached the daily goal and the remaining 6 participants reached the goal almost on half of the days. No subject asked to change the goal during the study period. The participant who met the goal every day said that he/she was not interested in increasing the goal due to the accomplishment feeling experienced by seeing that the goal was achieved every day. When asked about the difficulty of the step goal, 4 participants reported that it was too high, as they could not reach the goal in (almost) any day. Three participants found the goal appropriate, as in challenging but achievable, considering that it requires an extra effort to be achieved, as "it comes not by itself". One participant found the goal very difficult in the beginning but it motivated him/her to become more active, and at the end of the 4 weeks it was actually easy to achieve. Two participants found the goal easy or very easy. Finally, 1 participant said that he/she did not look at the goal during the 4 weeks. Most participants reported that the Activity Coach helped them to become more aware of their physical behavior and helped them to become more active.

"In the beginning I found the daily goal very high, but now it does not look much at all. If you walk 2

kilometers you are almost there. And then if you walk a bit in the house you reach the goal."

(Male, 66 yrs)

When comparing the measured average number of steps of each participant with the sample average, 4 participants are substantially above the average, 2 slightly above (less than 500 steps difference) and 4 
Table 2: Descriptive analysis of the parameters assessed regarding physical activity and emotional wellbeing.

\begin{tabular}{ll}
\hline Characteristic & Mean (standard deviation), range \\
\hline Study duration in days $(\mathrm{N}=292)$ & $27(1.5), 23-28$ \\
\hline Physical Activity $(\mathrm{N}=273)$ & \\
Steps & $6316(3688), 224-20158$ \\
$\quad$ Full sample & $2989-10572,(1554-5044), 224-20158$ \\
$\quad$ Variation between subjects & $4.51(2.67), 0.15-15.51$ \\
Distance $(\mathrm{km})$ & $1269(72), 1037-1440$ \\
Sedentary minutes per day & $29(34), 0-215$ \\
Moderate-to-intense active minutes per day & \\
\hline Daily wellbeing $(\mathrm{N}=272)$ & $5.53(0.79), 3-7$ \\
Joy & $5.55(0.77), 3-7$ \\
Awe & $5.63(0.72), 3-7$ \\
Interest & $5.64(0.90), 2-7$ \\
Serenity & $5.75(0.84), 3-7$ \\
Love / Friendliness & $5.68(0.92), 3-7$ \\
Amusement & $33.76(4.34), 19-42$ \\
Sum of positive emotions &
\end{tabular}

participants are below the average. When asked about how the participants perceive their physical activity level compared to their peers, two participants answered that they perceive themselves as more active than average, whereas, in fact, their measured physical activity is below average.

There are also divergences when looking at the comparison between self-perceived and objectively measured change in physical activity during the 4 weeks period. Three participants perceived themselves as becoming more active while in fact this did not happen, while 2 participants said they did not become more active while that data actually shows they did.

All participants were satisfied with the possibility to see an overview of the number of steps per day and per week. This overview helped participants becoming more aware of their physical activity encouraging them to become more active.

"It just motivates you, and I kind of like it to keep track of what you're doing and what you're doing per week and per day, yes I like it very much." (Female, 70) or "Yes, one time I was like 'ehm, today I had a little less, so tomorrow I should be moving a little more" (Female, 70)

Besides the functionalities currently available, participants would like to see an overview of their steps over longer periods of time (e.g. months or years) and would like to also see the distance performed on each day. Participants would also like to see personalized recommendations on how to achieve the desired physical activity level.
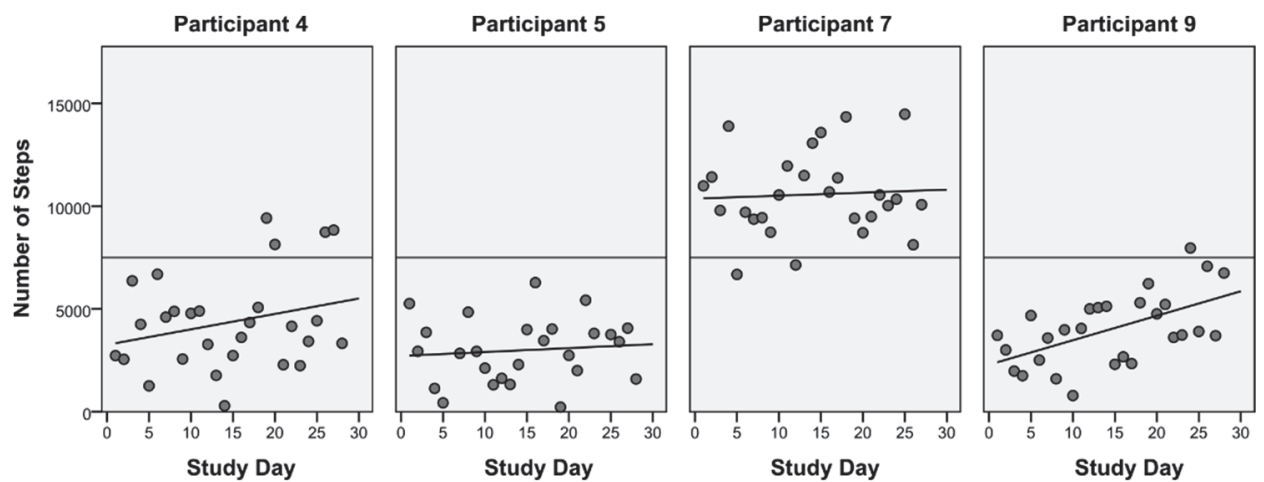

Figure 1: Variation of the number of steps per day for 4 participants. The coloured circles represent the number of steps taken on that day, the black line, the trend on the number of steps during the study. The green line represents the goal set to 7500 steps per day. 
Nine participants reported that they see the added value of using technology in daily life for monitoring physical activity. One of the participants bought a step counter for him/herself while participating in the study. Two participants said that, although it was fun to monitor physical activity, they would not do it in everyday life. These were the most active participants.

"I have to admit that, now that I have participated in this study, it is very clear for me how important it is to monitor physical activity." (Female, 73)

\subsection{Daily Monitoring of Positive Emotions}

From the small standard deviations of the ratings of experience of positive emotions in Table 2, it is visible that most of the participants use only 3 values of the Likert's scale providing a very small variability during the study. The reliability of the scale of positive emotions in this sample was acceptable (Cronbach's $\alpha=.93$ ).

The opinions of the participants regarding monitoring of emotional wellbeing varied notably. Six participants considered that they became more aware of their wellbeing by answering the questions.

"You really learn to realize the way you are and the way you work, you start to think about these things a little more, yes." (Female, 70)

However, only 4 participants saw an added value on this. Most participants were very critical about answering the wellbeing-related questions. The most referred remarks concerned the repetition of the questions, i.e. every day the same questions, and the fact that no feedback was provided.

"Ok but it is always the same question, then I answer every time the same, 6, 6, 6, 6 and ok that time a 7 because I was really happy, but nothing else..." (Male, 78)

"I mean I would consider a 4 too little, that is not correct. Then I have to choose among the other numbers, I don't stay at the 7, because that would be idiotic, right? No, I wouldn't do it!' (Female, 67)

Also, participants found it difficult to understand how the emotions differ between each other.

"The questions are in fact almost the same. If you are satisfied, than you are also happy more often, this type of thing..." (Female, 72)

One subjected mentioned that he/she would like to see the feedback on positive emotions linked to the feedback on physical activity.

\subsection{Relation Between Physical Activity and Positive Emotions}

The reports of the participants on the experience of monitoring positive emotions in daily life, made us question the reliability of the data collected and, consequently, limit the analysis to correlation analysis, only to grasp a feeling of the data. Table 4 provides the results of the bivariate relation between distinct ways of operationalize physical activity (i.e. steps, distance, and total number of minutes engaged in sedentary, light intensity and moderate to high intense physical activity) and positive emotions (happiness, cheerful, curious, calm, friendly and

Table 3: Self-perception of physical activity (PA) level compared to peers and objectively measured, as well as self-perception of change in physical activity during the 4 weeks of study and objectively measured. In grey shadow are represented the cases when the subjective perception and the measured data coincide.

\begin{tabular}{|c|c|c|c|c|}
\hline Participant & $\begin{array}{l}\text { Self-perception of PA } \\
\text { compared to peers }{ }^{\text {a) }}\end{array}$ & $\begin{array}{l}\text { Objectively measured } \\
\text { PA compared to peers } b)\end{array}$ & $\begin{array}{l}\text { Self-perception } \\
\text { of change in PA }\end{array}$ & $\begin{array}{l}\text { Objectively measured } \\
\text { change in PA }\end{array}$ \\
\hline 1 & + & + & - & + \\
\hline 2 & + & + & + & - \\
\hline 3 & - & - & + & - \\
\hline 4 & + & - & + & + \\
\hline 5 & I & - & - & I \\
\hline 6 & + & ++ & - & 1 \\
\hline 7 & + & ++ & - & I \\
\hline 8 & + & - & - & + \\
\hline 9 & - & - & + & - \\
\hline 10 & + & ++ & + & + \\
\hline 11 & + & ++ & 1 & I \\
\hline+ & \multicolumn{4}{|c|}{$\begin{array}{l}+ \text { represents more active than peers, / corresponds to neutral or unclear statements and - corresponds to less active than } \\
\text { peers }\end{array}$} \\
\hline+ & \multicolumn{4}{|c|}{$\begin{array}{l}++ \text { represents more active than peers and quite above the daily goal, }+ \text { more active than peers but average below goal line, } \\
\text { - less active than peers }\end{array}$} \\
\hline
\end{tabular}


satisfied, and sum value). The number of steps per day and the distance is significantly associated to the daily ratings of curiosity/interest $(\mathrm{p}<0.05)$. Furthermore, the number of sedentary minutes is associated to the rating of friendliness $(p<0.05)$.

\section{DISCUSSION}

In this study we compared the subjective and objective experience of coaching physical activity and monitoring positive emotions in daily life using mobile technology. Older adults see an added value on monitoring physical activity, but not so much in monitoring wellbeing. Moreover, we investigated the relation between physical activity and six discrete positive emotions. Our results suggest that the relation between physical activity and positive emotions is not direct, suggesting that other factors might act as moderators. However, due to the small sample size and considering the criticism concerning the assessment of positive emotions, these results should be taken with caution and we highly encourage further research. In the following subsections, the results associated to each one of the objectives are discussed in more detail.

\subsection{Experience of Promotion of Physical Activity in Daily Life}

In general, the participants were very satisfied with the opportunity to monitor physical activity in daily life and, in particular, with the tracker chosen. This physical activity tracker is discrete, can be worn in the pocket, has long battery duration and is simple. This fact suggests that, although older adults are often not the target population of the market of physical trackers, after a small nudge to start using the technology, they actually perceive an added value.

The average number of steps of the full sample was approximately 6300 steps/day, with large individual differences. In the present moment there is no commonly accepted guideline for the number of steps older adults should take per day. Literature elsewhere reports similar ranges of steps with healthy older adults ranging from 2000 to 9000 steps/day and special populations 1200 to 8800 steps/day (TudorLocke et al. 2011). It is therefore not surprising, that some participants experienced the goal of 7500 steps/day as difficult, while others reached it with no difficulty every day. This large variability in the daily number of steps emphasizes the need for tailored interventions with goals set specifically to each individual. A possible approach to automatically goal-setting is provided by (Cabrita et al. 2014). The Goal-Setting theory suggests that, to be motivating, goals must be challenging but achievable (Locke \& Latham 2002). This is clearly seen in the subjective experience reported by the participants in the interview. Those who were already very active, and constantly above the daily goal, reported limited added value from the system; on the contrary, those who started below the goal, but close to it, mentioned that the system helped to make them more aware of their lack of physical activity and motivated them to become more active. Similar results are presented by (Eisenhauer et al. 2016).

During the four weeks of study, several technical issues were reported, on the connectivity between the step counter and the smartphone. Similar technical problems are also reported in literature (Harrison et al. 2014). Despite these issues, the participants reported a positive experience, perceived the

Table 4: Bivariate analysis (Spearman 2-tailed) between several measures of physical activity and positive emotions.

\begin{tabular}{|c|c|c|c|c|c|c|c|}
\hline & Joy & Awe & Interest & Serenity & Love & Amusement & $\begin{array}{c}\text { Sum } \\
\text { Positive } \\
\text { Emotions }\end{array}$ \\
\hline steps & .056 & .099 & $.141^{*}$ & -.053 & -.099 & .025 & .020 \\
\hline distance & .049 & .095 & $.138^{*}$ & -.046 & -.090 & .028 & .021 \\
\hline \#minutes inactive & .000 & -.056 & -.021 & .093 & $.152 *$ & .014 & .045 \\
\hline $\begin{array}{l}\text { \#minutes lightly } \\
\text { active }\end{array}$ & -.001 & .041 & -.010 & -.071 & -.111 & -.001 & -.035 \\
\hline $\begin{array}{l}\text { \#minutes moderate- } \\
\text { to-active }\end{array}$ & -.010 & .017 & .057 & -.072 & -.119 & -.035 & -.052 \\
\hline
\end{tabular}

$* \mathrm{p}<0.05$. 
technology as useful, and were comfortable using it. Furthermore, it is difficult to distinguish between inactive time and not worn time. This is particularly difficult in the evenings, as it might be that older adults stop carrying the step counter but keep doing their normal activities.

\subsection{Daily Monitoring of Emotional Wellbeing}

The opinions about monitoring emotional wellbeing diverged. While part of the participants became more aware about their wellbeing, only a few perceived an added value. The strongest criticism was that participants did not receive any feedback on their answers. Some participants also referred that they were not used to reflect on their emotions and do not feel comfortable doing so. Participants perceived wellbeing, or mental health, as something too personal to provide information about to a machine. This is perceived differently than information related to physical health, showing the stigma might still be present when talking about mental health.

Regarding the assessment method, participants perceived the questions as too repetitive. We suggest that, while experience sampling is a promising method to assess emotions, attention should be given when designing the questions. For example, variation in the phrasing should be considered to avoid unreflective answers.

Further research should be performed on the assessment of emotional wellbeing in daily life. One can think of strategies as facial recognition or text mining from the data in social media; however, these methods do not request reflection from the person being assessed, as initially desired in this study. Nevertheless, predictive models of daily emotions are currently being investigated and can open room for interventions in daily life, yet with limited confidence in positive results (Asselbergs et al. 2016).

\subsection{Relation Between Physical Activity and Positive Emotions}

Our study follows the 3 recommendations of the Kanning and colleagues for within-subject analysis of physical activity and affective states: objective assessment of physical activity, the importance of real time assessment, affective states measured electronically (Kanning et al. 2013). Despite following these recommendations, we were not able to investigate the dynamics between positive emotions and physical activity, as initially desired. Based on the interviews performed after the study, we considered that the answers given to the daily ratings of positive emotions were not reliable. In any case, our preliminary analysis suggests that there is some evidence to confirm a relation between positive emotions and physical activity, dependent on the operationalization of the outcome. We recommend further research investigating the context of the activities. Another suggestion is to perform studies for longer periods of time and extract only the data points in which the experience of positive emotions deviates from the mode value.

\section{CONCLUSION}

Our study suggests that older adults are willing to monitor their physical activity in daily life and that the technology helps them becoming more aware of their current activity level. On the contrary, older adults perceive limited added value of monitoring emotional wellbeing in daily life - in this study operationalized as experience of positive emotions mostly due to the repetitiveness of the questions. The interviews performed with the participants at the end of the study revealed low reliability on the data collected on the wellbeing. For this reason, a thorough analysis on the relation between physical activity and positive emotions was not performed.

Further research needs to be performed in the mobile assessment of emotional wellbeing before being able to look at the relations with other factors. The interviews performed after using technology were extremely important to let us make sense of the data collected. We would like to alert researchers using mobile assessment of emotions to question the reliability of their data when repeating the same questions for a long period of time.

\section{ACKNOWLEDGEMENTS}

The work presented in this paper is being carried out within the PERSSILAA project and funded by the European Union 7th Framework Programme under Grant FP7-ICT-610359. The authors would like to thank Jandia Melenk and Nada El Meshawy for their support conducting the interviews.

\section{REFERENCES}

Alley, S. et al., 2016. Interest and preferences for using advanced physical activity tracking devices: results of a national cross-sectional survey. BMJ Open, 6(7), p.e011243. Available at: http://dx.doi.org/10.1136/ bmjopen-2016-011243. 
Asselbergs, J. et al., 2016. Mobile Phone-Based Unobtrusive Ecological Momentary Assessment of Day-to-Day Mood: An Explorative Study. Journal of Medical Internet Research, 18(3), p.e72. Available at: $\mathrm{http} / / / \mathrm{dx}$.doi.org/10.2196/jmir.5505.

Braun, V. \& Clarke, V., 2006. Using thematic analysis in psychology. Qualitative Research in Psychology, 3(May 2015), pp.77-101.

Cabrita, M. et al., 2014. Automated Personalized Goalsetting in an Activity Coaching Application. In Proceedings of the 3rd International Conference on Sensor Networks. SCITEPRESS - Science and and Technology Publications, pp. 389-396. Available at: http://dx.doi.org/10.5220/0004878703890396.

Case, M.A. et al., 2015. Accuracy of smartphone applications and wearable devices for tracking physical activity data. Jama, 313(6), pp.625-626. Available at: http://dx.doi.org/10.1001/jama.2014.17841.

Csikszentmihalyi, M. \& Hunter, J., 2003. Happiness in everyday life: The uses of experience sampling. Journal of Happiness Studies, 4(January), pp.185-199. Available http://dx.doi.org/10.1023/A:1024409732742.

Eisenhauer, C.M. et al., 2016. Acceptability of mHealth Technology for Self-Monitoring Eating and Activity among Rural Men. Public health nursing. Available at: http://www.ncbi.nlm.nih.gov/pubmed/27757986.

Ferguson, T. et al., 2015. The validity of consumer-level, activity monitors in healthy adults worn in free-living conditions: a cross-sectional study. The international journal of behavioral nutrition and physical activity, 12, p.42. Available at: http://dx.doi.org/10.1186/ s12966-015-0201-9.

Fredrickson, B.L., 2013. Positive Emotions Broaden and Build. In P. Devine \& A. Plant, eds. Advances in Experimental Social Psychology. Burlington: Academic Press, pp. 1-53.

Fredrickson, B.L., 2001. The role of positive emotions in positive psychology: The broaden-and-build theory of positive emotions. American Psychologist, 56(3), pp.218-226.

Harrison, D. et al., 2014. Tracking physical activity: problems related to running longitudinal studies with commercial devices. Proceedings of the 2014 ACM International Joint Conference on Pervasive and Ubiquitous Computing Adjunct Publication - UbiComp '14 Adjunct, pp.699-702. Available at: http://dx.doi.org/10.1145/2638728.2641320.

Kanning, M.K., Ebner-Priemer, U.W. \& Schlicht, W.M., 2013. How to Investigate Within-Subject Associations between Physical Activity and Momentary Affective States in Everyday Life: A Position Statement Based on a Literature Overview. Frontiers in psychology, 4(April), p.187. Available at: http://dx.doi.org/10.3389 /fpsyg.2013.00187.

Kooiman, T.J.M. et al., 2015. Reliability and validity of ten consumer activity trackers. BMC sports science, medicine and rehabilitation, 7, p.24. Available at: http://dx.doi.org/10.1186/s13102-015-0018-5.
Lee, J.M., Kim, Y. \& Welk, G.J., 2014. Validity of consumer-based physical activity monitors. Medicine and Science in Sports and Exercise, 46(9), pp.1840 1848.

Locke, E. a. \& Latham, G.P., 2002. Building a practically useful theory of goal setting and task motivation: A 35year odyssey. American Psychologist, 57(9), pp.705717. Available at: http://dx.doi.org/10.1037/0003066X.57.9.705.

Lyubomirsky, S., King, L. \& Diener, E., 2005. The benefits of frequent positive affect: does happiness lead to success? Psychological bulletin, 131(6), pp.803-55. Available at: http://dx.doi.org/10.1037/0033-290.131 .6 .803 .

Nelson, M.B. et al., 2016. Validity of Consumer-Based Physical Activity Monitors for Specific Activity Types. Medicine \& Science in Sports \& Exercise, (March).

Steverink, N. et al., 2001. Measuring frailty: developing and testing the GFI (Groningen Frailty Indicator). Gerontologist, 41, p.236.

Sun, F., Norman, I.J. \& While, A.E., 2013. Physical activity in older people: a systematic review. BMC public health, 13(1), p.449. Available at: http://www .biomedcentral.com/1471-2458/13/449.

Tabak, M., op den Akker, H. \& Hermens, H., 2014. Motivational cues as real-time feedback for changing daily activity behavior of patients with COPD. Patient education and counseling, 94(3), pp.372-8. Available at: http://dx.doi.org/10.1016/j.pec.2013.10.014.

Tudor-Locke, C. et al., 2011. How many steps/day are enough? For older adults and special populations. The international journal of behavioral nutrition and physical activity, 8(1), p.80. Available at: http://www.ijbnpa.org/content/8/1/80.

Tully, M.A. et al., 2014. The validation of Fibit ZipTM physical activity monitor as a measure of free-living physical activity. BMC Res Notes, 7, p.952. Available at: http://dx.doi.org/10.1186/1756-0500-7-952.

Ware, J.E., 1993. SF-36 Health Survey: Manual and interpretation guide, Boston, MA: The Health Institute, The New England Medical Center.

Wolvers, M. et al., 2015. Effectiveness, Mediators, and Effect Predictors of Internet Interventions for Chronic Cancer-Related Fatigue: The Design and an Analysis Plan of a 3-Armed Randomized Controlled Trial. JMIR Research Protocols, 4(2), p.e77. Available at: http://dx.doi.org/10.2196/resprot.4363.

World Health Organization, 2009. Global Health Risks: Mortality and burden of disease attributable to selected major risks. Bulletin of the World Health Organization, 87, pp.646-646. Available at: http://www.who.int /healthinfo/global_burden_disease/GlobalHealthRisks _report_full.pdf. 